\title{
Design and Development of a High-Throughput System for Learning and Memory Research on Zebrafish
}

\author{
Hamed Hanafi Alamdari1,2*, Nancy Kilcup ${ }^{1,2^{*}}$, Zachary Ford ${ }^{3}$, Florentin Wilfart ${ }^{1,2,4}$, \\ David C. Roach ${ }^{2}$, Michael Schmidt ${ }^{1,4}$
}

\author{
${ }^{1}$ School of Biomedical Engineering, Dalhousie University, Halifax, Canada \\ ${ }^{2}$ Rowe School of Business, Dalhousie University, Halifax, Canada \\ ${ }^{3}$ Department of Medical Sciences, Dalhousie University, Halifax, Canada \\ ${ }^{4}$ Department of Anaesthesia, Dalhousie University, Halifax, Canada \\ Email: hamed.hanafi@dal.ca
}

How to cite this paper: Alamdari, H.H., Kilcup, N., Ford, Z., Wilfart, F., Roach, D.C. and Schmidt, M. (2018) Design and Development of a High-Throughput System for Learning and Memory Research on Zebrafish. Journal of Behavioral and Brain Science, 8, 351-368.

https://doi.org/10.4236/jbbs.2018.86023

Received: April 21, 2018

Accepted: June 9, 2018

Published: June 12, 2018

Copyright $\odot 2018$ by authors and Scientific Research Publishing Inc. This work is licensed under the Creative Commons Attribution International License (CC BY 4.0).

http://creativecommons.org/licenses/by/4.0/

\begin{abstract}
Background: Since 2004, zebrafish have become the state-of-the-art, in vivo model for biomedical research due to their genetic and physiological homology with humans, inexpensive high-quantity breeding, and quick development in a highly-controlled environment suitable for longitudinal studies. New Method: To fully utilize the zebrafish model, a novel, automated, high-throughput system was designed. Shoals of five zebrafish were placed in 16 tanks and automatically fed over two days for a total of 16 training sessions. Color LED lights were used as the stimulus for each shoal coinciding with the release of food for a duration of 20 seconds. This system was tested on two age groups: 6- and 11-month-old. Results: After three training sessions, the median height of the school in the tank during stimulus was significantly higher than that of the naive fish during the first training session. All subsequent training sessions demonstrated similar behaviour. A decline in memory retention, as defined by a reduction in the median height during light stimulus (i.e. no simultaneous food delivery), was observed 8 days post training. Comparison with existing methods: The high-throughput nature of this system allows for simultaneous training of 16 tanks of fish under identical conditions without human interaction and provides a means to rapidly assess their learning and memory behaviours. Conclusion: Results provide a baseline for understanding the normal cognitive processes of learning and memory retention in zebrafish. This work paves the way for future studies on the impacts of therapeutic agents on these cognitive processes.
\end{abstract}

${ }^{\star}$ Co-first authors contributed equally to this work. 


\section{Keywords}

Learning, Behavior, High Throughput System, Automation, Cognition

\section{Introduction}

Since 2004, zebrafish (Danio rerio) usage in biomedical research has increased significantly [1]-[6]. Use of zebrafish as a biomedical research model saw an increase from $7 \%$ of all animal studies in 2004 to $15 \%$ by 2013 [3]. This is largely due to their high genetic homology to humans [7] [8] and ease of breeding, making zebrafish an excellent animal model for translational research. The breadth of topics investigated using zebrafish includes neurological [6] [9] [10] genetic [11] [12], kidney [13] [14], and, liver [15] diseases. Furthermore, zebrafish are frequently used to evaluate the effect of various therapies and/or treatments on cognition and behaviour [4] [16] [17] [18]. The potential applications for using zebrafish continue to grow and expand.

Until recently, two of the most common animal models used in biomedical research were roundworms (Caenorhabditis) and mice (Mus musculus) [3]. Roundworms provide less complex behaviour and more straightforward gene interaction [3], but their neurochemistry is highly simplistic. Conversely, mice provide high neurochemical complexity [19], but their slow reproductive rates impede genealogical studies [20]. Zebrafish combine the benefits of both mouse and roundworm models, as they possess similar sophisticated sensory and motor systems to mice and have rapid reproduction rates similar to roundworms [9] [19] [21] [22] [23]. In addition, zebrafish have a longer average lifespan than both C. elegans and M. musculus [24] [25], making them an excellent choice for longitudinal studies. Furthermore, breeding and aging of zebrafish is conducted in a precisely controlled environment, minimizing potential discrepancies caused by environmental variations. This allows for execution of controlled studies with hundreds of zebrafish over short time spans at a manageable cost.

Despite the high level of understanding of zebrafish neurochemistry and gene interaction, studies regarding zebrafish learning and memory are inconclusive and the specifics of these behaviours remain unknown [26] [27]. Protocols for zebrafish learning are still unrefined [27] and require extensive manual handling. Therefore, rapid, reliable, and reproducible learning and memory paradigms must be developed and standardised in order to further ongoing zebrafish research in this area.

To expand the use of the zebrafish model in cognitive studies a better understanding of their normal behaviour is needed. Few long-term learning and memory retention studies on zebrafish have been published [28]-[34]. Many of these studies observe the memory of larval zebrafish [32] [33] [34], whose long-term retention is significantly shorter than that of adult zebrafish [27] [28] [35]. One method used to evaluate zebrafish behaviour is to monitor changes to 
their shoaling behaviour [22] [26] [36]. The nature of this model requires zebrafish to be housed and trained individually, which can have negative impacts on zebrafish [4] [37] [38] including detrimental neurophysiological effects [17], increased aggression [39] [40] [41], and the development of metabolic diseases [17] [42] [43] [44]. Modifications to both the training protocols and methods of analysis are needed that will allow for better assessment of behaviour in a non-stressful environment that better reflect the normal conditions for the fish.

Recent improvements to learning paradigms include automatic feeders and the use of video recording equipment. These additions eliminate the unintentional biases caused by the presence of an experimenter during training and testing [22] [45] [46]. Recently, a system has been developed that uses automated tracking software to analyze fish movements [46] [47]. However, the training and testing processes are extremely labour intensive since a maximum of three tanks can be trained at one time [33]. Another limitation is that training and testing is done in uncirculated water, which may cause stress to the fish [48]. Hence, an opportunity exists to develop high-throughput learning systems where fish can be fed automatically and behaviour can be recorded on video for subsequent analysis. In order to streamline learning and memory retention studies in a large scale system, a novel training and testing system equipped with a video monitoring system is needed.

This study presents an efficient, automated, high-throughput fish housing and monitoring system isolated from human interference for the assessment of learning and memory retention of zebrafish.

\section{Materials and Methods}

\subsection{Fish Husbandry}

\subsubsection{Test Species}

Wild-type AB strain zebrafish (Danio rerio), of both sexes, were used in this study. Adult animals were obtained from Dalhousie University (Halifax, NS, Canada) as two age groups of 6 and 11 months. After completion of the behavioural study, subjects were euthanized by immersion in unbuffered $10 \%$ aqueous tricaine methanesulfonate (MS-222).

\subsubsection{Test Housing}

Subjects were placed into $3 \mathrm{~L}$ tanks $(\mathrm{n}=16)$ at a housing density of 5 fish $(\mathrm{m}=5)$ per tank. The water quality was controlled using a continuous recirculating system with flow rates adapted to the flow rates on the breeding and storage racks of the large system. Water quality was monitored daily and adjusted when necessary. Fish were maintained at $26^{\circ} \mathrm{C} \pm 2{ }^{\circ} \mathrm{C}$ with a pH $7.56 \pm 0.3$ and a conductivity of $800 \pm 30 \mu \mathrm{S}$ under a 14:10 h light:dark photoperiod. During training periods, fish were fed an artificial replacement diet (Golden Pearl Reef 100 - 200 $\mu \mathrm{m}$, Brine Shrimp Direct) eight times daily via an automatic feeding system and during retention periods, fed the same replacement diet manually twice daily. 


\subsubsection{Animal Ethics}

All procedures were reviewed and approved by the Dalhousie University Committee on Laboratory Animals (14-141) and conducted in accordance with the Canadian Council on Animal Care (CCAC) Guidelines on the care and use of experimental animals (1993) [49].

\subsection{Video Acquisition}

\subsubsection{Observation System}

A custom-made fish observation system was designed (Figure 1) for simultaneous surveillance of 16 fish tanks. Figure 1 represents only one half of the system with the other half being a mirror image of the system shown. The system has three major components: 1 ) the water system; 2) the automated training and retention testing control system; and 3) the video acquisition system.

The water system includes a large insulated reservoir equipped with a filter, biological filter media in a net, aquarium heater, and an aerator connected to an air pump and water pump. Water was pumped through $3 / 4$ " potable water pipes and delivered to the tanks using $1 / 8$ " tubes connected to the main pipes. Flow was balanced using ball valves with a T-joint and a $1 / 8$ " push-fitting. Tank drain pipes were fitted into 1" standpipes to drain back into the reservoir. Water was

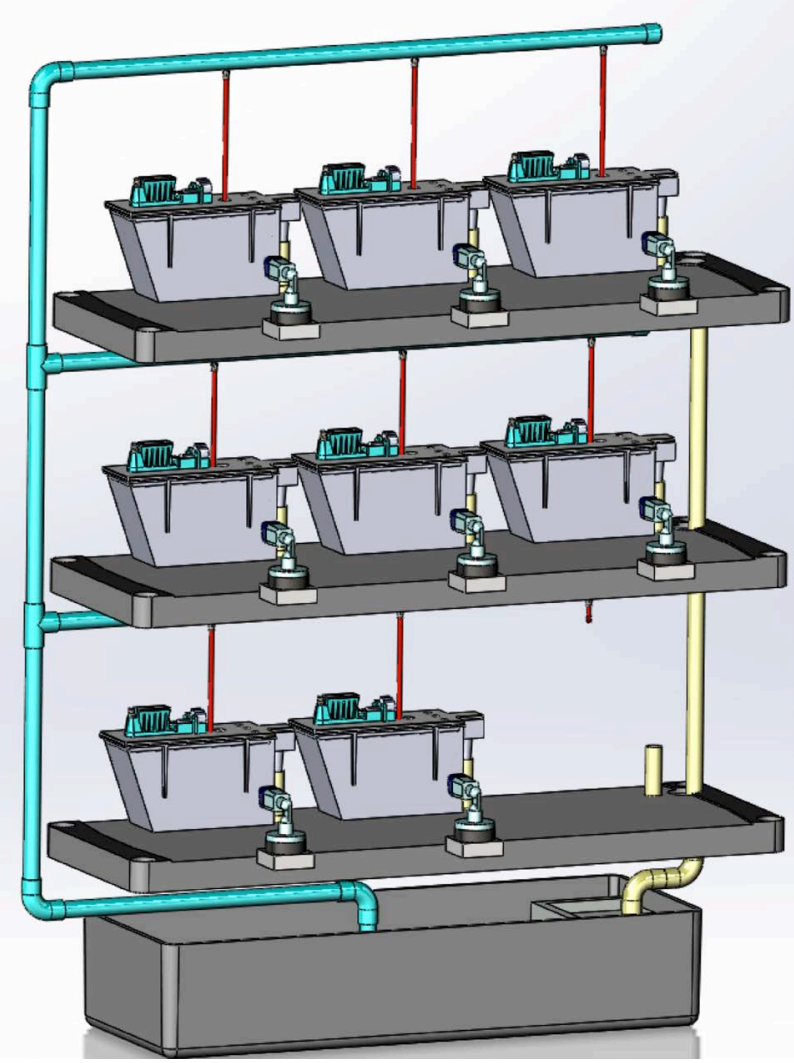

Figure 1. Schematic of the test set up of one side of the symmetrical water system with the automated training and retention system, and video acquisition system. 8 tanks of 16 shown with tubing and video acquisition hardware for each testing unit. Rendered using 3D CAD design software (SolidWorks, Waltham, MA, USA). 
continuously flowing through the system with an adjustable flow rate set by both the inlet and outlet valves installed at each level.

The automated training and retention system composes a custom control system, two sets of lights (SMD5050, RioRand, Richmond, BC, Canada), and a feeder system. A microcontroller board (Mega, Arduino, New York, NY, USA) is the heart of the control system with relays controlling the RBG light strips which are connected to the feeder system using network cables. During training, food delivery and green light exposure was triggered simultaneously with a timer. The timer triggers the Arduino to rotate the stepper motors (28BYJ-48, Keyes, Shenzhen, China) on the feeders and turn on the RGB LED lights set to green. A second timer, independent of the Arduino, was used to control the overhead daylight color LED lights used to mimic the light:dark photoperiod. The components of the feeding system include a 3D-printed feeder body (Nylon, Makerbot Replicator 2, Dalhousie University) with a food reservoir, a step motor with shield, and a $5 \mathrm{~mm}$ stainless steel drill bit that rotates to deliver the food.

Zebrafish movements were recorded using a security camera system (720p HD Weatherproof Night Vision, Lorex, Markham, Canada). Each tank has a dedicated camera placed $17 \mathrm{~cm}$ from the tank wall so that the camera is perpendicular to the tank wall. Video was recorded at a frame rate of $30 \mathrm{fps}$ and stored on the Lorex data logging system. The infrared sensor on the cameras was blocked in order to prevent influence on fish behaviour.

\subsubsection{Training Paradigm}

Doyle et al. (2017) demonstrated that a light signal can be associated with the release of food as a conditioned stimuli [46]. In this paper, during training, fish were simultaneously fed by the automatic feeder and exposed to green light (stimulus) for 20 seconds via a green LED light strip installed at the bottom front edge of the tank (Figure 2). Over a two-day period of training, this process was repeated 16 times during the daylight cycle ( 8 times in each of the two-day training). During memory retention testing, fish were exposed to the same green light, however, no food was provided. The subject's ability to retain this training was evaluated by testing behavioural changes when light stimulus was provided without being paired with food. Memory retention testing was performed and recorded with 6-month subjects (young) at 2-, 11-, 18-, 25-, and 28-day time points and 11-month subjects (old) at 2-, 8-, 14-, 28-, and 35-day time points after the initial (two-day) training period. This baseline memory retention behaviour of the zebrafish was evaluated for 160 subjects.

\subsection{Post-Acquisition Video Analysis}

The widely used, open-source tracking software Ctrax (California Institute of Technology, Pasadena, CA, USA) was originally created to track group movements of fruit flies (Drosophila) [50]. The native software only tracks the movement of fruit flies on a planar surface and is unable to accurately track zebrafish when swimming in three dimensions. The rigid exoskeleton of the fruit fly 




Figure 2. Schematic of a single tank set up with feeding and video acquisition systems in place and axes for 2D video tracking. Rendered using 3D CAD design software (SolidWorks, Waltham, MA, USA).

means that their shape is constant and independent of the direction in which they fly. Contrary to this, zebrafish have a non-rigid endoskeleton that changes shape depending on the direction they are swimming and the way they are facing the camera, which makes tracking more difficult. However, taking advantage of the shoal housing and analyzing their behaviour as a social group, Ctrax can be utilized to track every fish in the tank continuously.

Most tracking algorithms function by detecting the non-moving objects using a total recording frame average and subtracting it from each frame to detect the moving objects. This is not a simple process for four reasons: 1) Banding artefacts due to the similarity in the sampling rate and frequency of the LED lights, the recordings showed continuously moving background banding artefacts. 2) Reflections of the fish visible when swimming close to the sides of the tank and surface of the water, giving inaccurate results from the tracking software. 3) Changing shape of fish while moving, especially when moving in the z-direction (in relation to the axes labeled in Figure 2). 4) Merging of subjects when two fish cross paths, making it nearly impossible to differentiate each individual fish after their paths have uncrossed. The specific challenges and their respective solutions are explained below.

\subsubsection{Video Editing}

The uncompressed digital video files (in ASF format) were transferred onto a flash drive (Lexar, Micron, Milpitas, CA, USA). The fish-eye effect was removed using defishr (v1, proDAD®, city, country, https://www.prodad.com/). The files were then exported in MP4 format. Edited video footage was then reviewed and 
trimmed to contain the 20 -second sections before and during subject exposure to the stimulus.

\subsubsection{Tracking Algorithm}

Quantification of fish motion was performed using the machine vision program, Ctrax (v0.5.6, Pasedena, CA, USA, http://ctrax.sourceforge.net/), as can be seen in Figure 3. This program was originally created to track group movement of fruit flies (Drosophila) [50] in a two dimensional space. Therefore, modifications to the native software settings were required in order to reliably track movement of zebrafish (Appendix 1). This was later verified with randomly selected video samples.

\subsubsection{Data Processing}

The output files prepared by Ctrax were analyzed in MATLAB $^{\circledR}$ (Version R2016a, MathWorks ${ }^{\circledR}$, Natick, MA, USA). The "Behavioural microarray" toolbox provided by Branson et al. (2009) [50] was modified based on the specific properties of tank videos to output motion parameters. Due to varying level of brightness in the tanks, a fish detected in a frame could be detected as a new fish in the next frame. This led to the output files tracking movements of more than five fish. Since every fish in the tank was detected at every frame, the median height of all detected fish was calculated as the tank's tracked movement (Figure 4).

\subsubsection{Statistical Analysis}

Obtained data for each session was first tested for normal distribution with Kolmogorov-Smirnov test $($ alfa $=0.01$ ) as well as the D'Agostino and Pearson omnibus test $(\mathrm{alfa}=0.01)$. Subsequently, data was evaluated using a one-way ANOVA followed by Sidak's post-hoc test. The statistical software Prism 7 (GraphPad Software, Inc., San Diego, CA, USA) was used for this purpose. In all comparisons, ${ }^{*} \mathrm{p}<0.02,{ }^{* *} \mathrm{p}<0.01,{ }^{* *} \mathrm{p}<0.001$ and ${ }^{* * *} \mathrm{p}<0.0001$ were used to indicate statistical significance. Values are presented as the mean \pm standard deviation $(\mathrm{SD})$.

\section{Results}

The median height $\left(\mathrm{Y}_{\mathrm{m}}\right)$ of both the old (Figure 5) and young (Figure 6) fish shoals were higher during exposure to stimulus in all training sessions (light + food) than the $\mathrm{Y}_{\mathrm{m}}$ for shoals in the 20 seconds prior to the stimulus. Using a one-way ANOVA, $\mathrm{Y}_{\mathrm{m}}$ during the pre-light phase was compared between all training sessions and retention tests; no statistical difference in $Y_{m}$ was observed.

Specifically comparing the $\mathrm{Y}_{\mathrm{m}}$ of old fish during the stimulus, observed in Figure 5, there was no significant difference between session $1\left(S_{1}\right)$ and session $\left(S_{2}\right)$. However, $Y_{m}$ of $S_{1}$ was significantly different from $S_{3}-S_{16}(p<0.001) . Y_{m}$ of $S_{1}$ was significantly different from retention sessions $R_{2}(p<0.0001)$ and $R_{8}(p<$ 0.02 ), but not different from $\mathrm{R}_{14}$.

The behaviour of the young fish during training sessions (Figure 6) mimicked that of the older fish (Figure 5). The $Y_{m}$ of young fish in $S_{1}$ was not significantly 




(a)

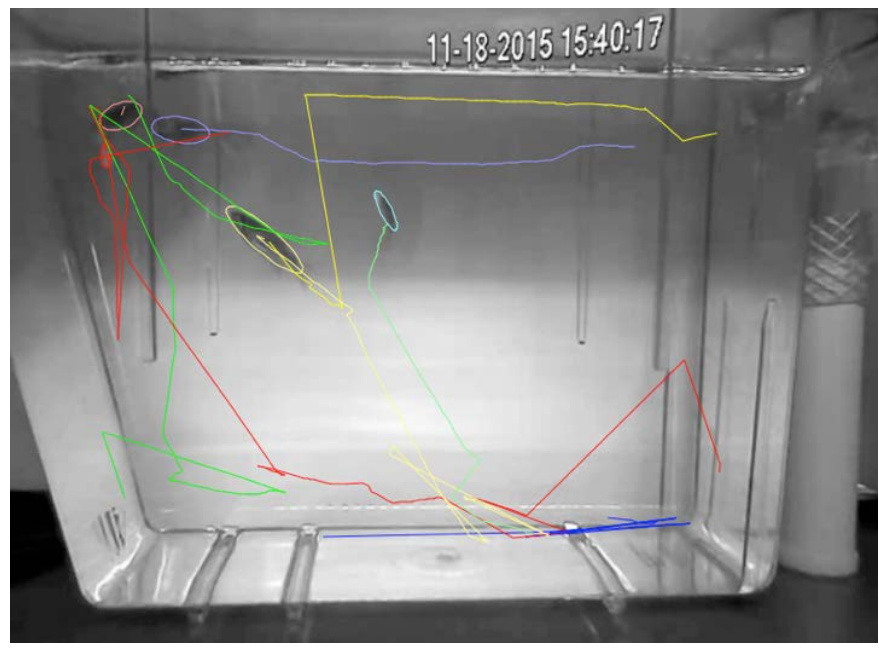

(b)

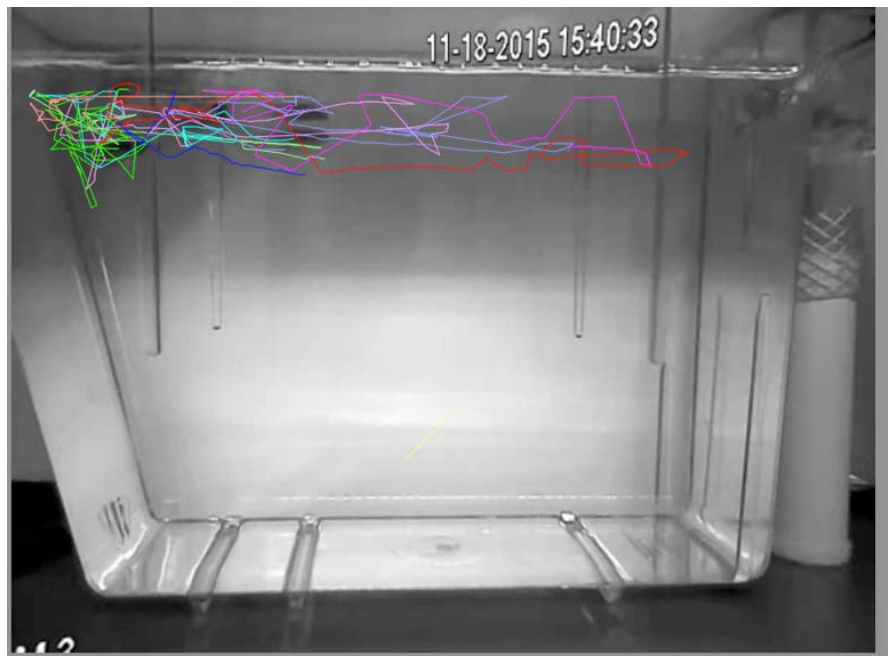

(c)

Figure 3. Tracking in a representative tank during light. Each fish is encircled by a unique color, corresponding with their pathway color. (a) Detecting fish from background; (b) First 100 frames of tracked fish movement; (c) Last 200 frames of tracked fish movement. 




Figure 4. Tracked fish height during light (black) and median of all fish height (blue) normalised to the height of the tank represented as a percentage. Top represents the top $20 \%$ of the water height (equivalent to the top 3 centimeters of water height) and is emphasized in light blue color.

\section{Median height of Old Fish Before and During Light}

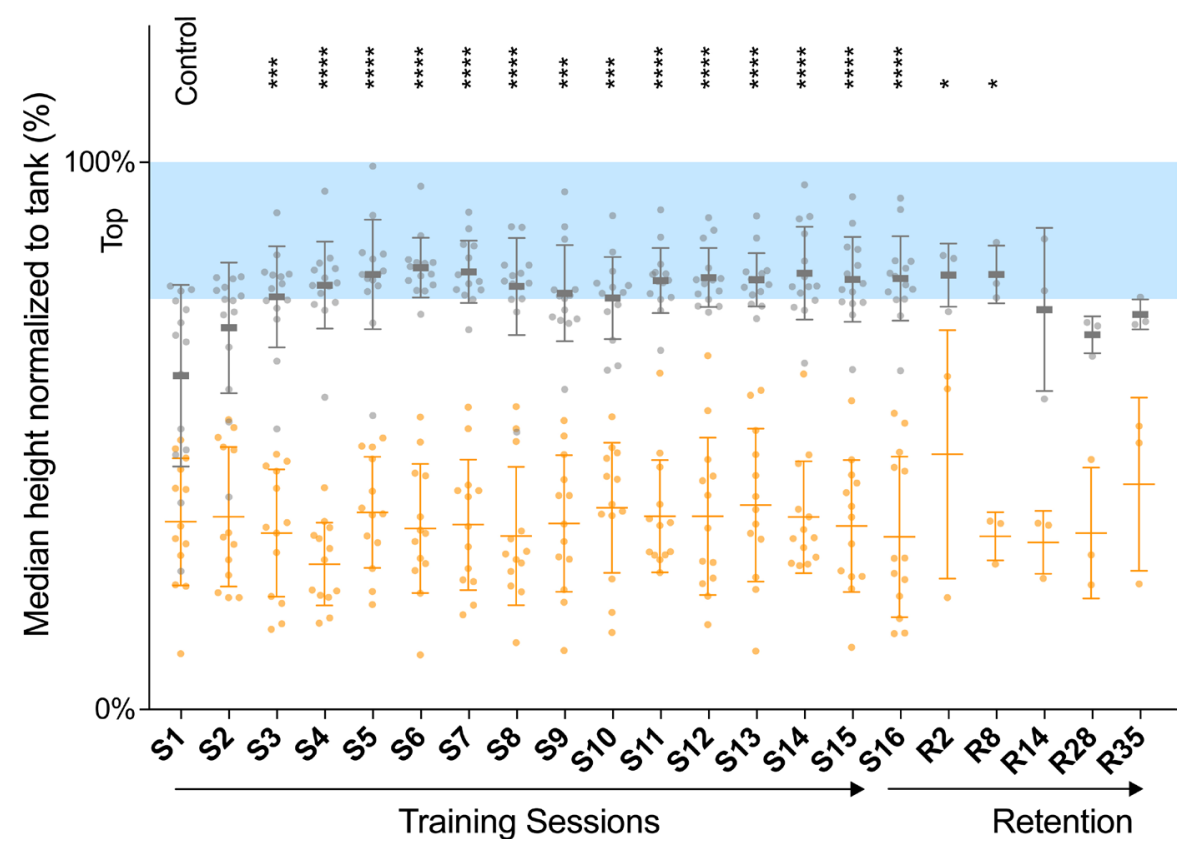

Figure 5. Median height of old fish before (yellow lines) and during (gray lines) the light stimulation. ${ }^{*}$ shows the statistical significance between $\mathrm{Y}_{\mathrm{m}}$ of a specific session with the control session $\left(\mathrm{S}_{1}\right)$ during the (light + food) stimulus where ${ }^{*},{ }^{* *},{ }^{* *}$ and ${ }^{* * *}$ represent $\mathrm{p}<0.02, \mathrm{p}<0.01, \mathrm{p}<0.001$ and $\mathrm{p}<0.0001$ respectively.

different from $S_{2}$. However, as observed in Figure 6, $Y_{m}$ of young fish in $S_{1}$ was significantly different from $S_{3}-S_{16}$. $Y_{m}$ of $S_{1}$ was only significantly different from 
retention session $R_{2}$, but not from $R_{11}-R_{28}$.

\section{Discussion}

Zebrafish have high genetic and physiological homology to humans and can be easily genetically manipulated for research. Their rapid development and their relatively long lifespan provide the opportunity for relevant and statistically powered longitudinal studies. These benefits, along with inexpensive easy breeding and the capability of group housing, have contributed to the zebrafish emerging as a new state-of-the-art investigational tool for biological and medical research [3]. Therefore, an efficient, high-throughput, and reproducible system was designed to fully utilize the potential of zebrafish as a toolset.

Recently, Doyle et al. (2017) presented a system for analysing zebrafish learning and memory. The authors demonstrated that classical conditioning is possible in zebrafish where either a visual or auditory signal can be associated with the release of food as a conditioned stimuli [46]. However, due to the limited number of tanks that can be trained and probed for memory retention at the same time (only three) makes this process lengthy and extremely labour intensive.

Following the example of Doyle et al., and implementing a philosophy of experimental refinement, a novel high-throughput system for analyzing zebrafish shoal behaviour was designed. This system minimized human interaction as well

\section{Median height of Young Fish Before and During Light}

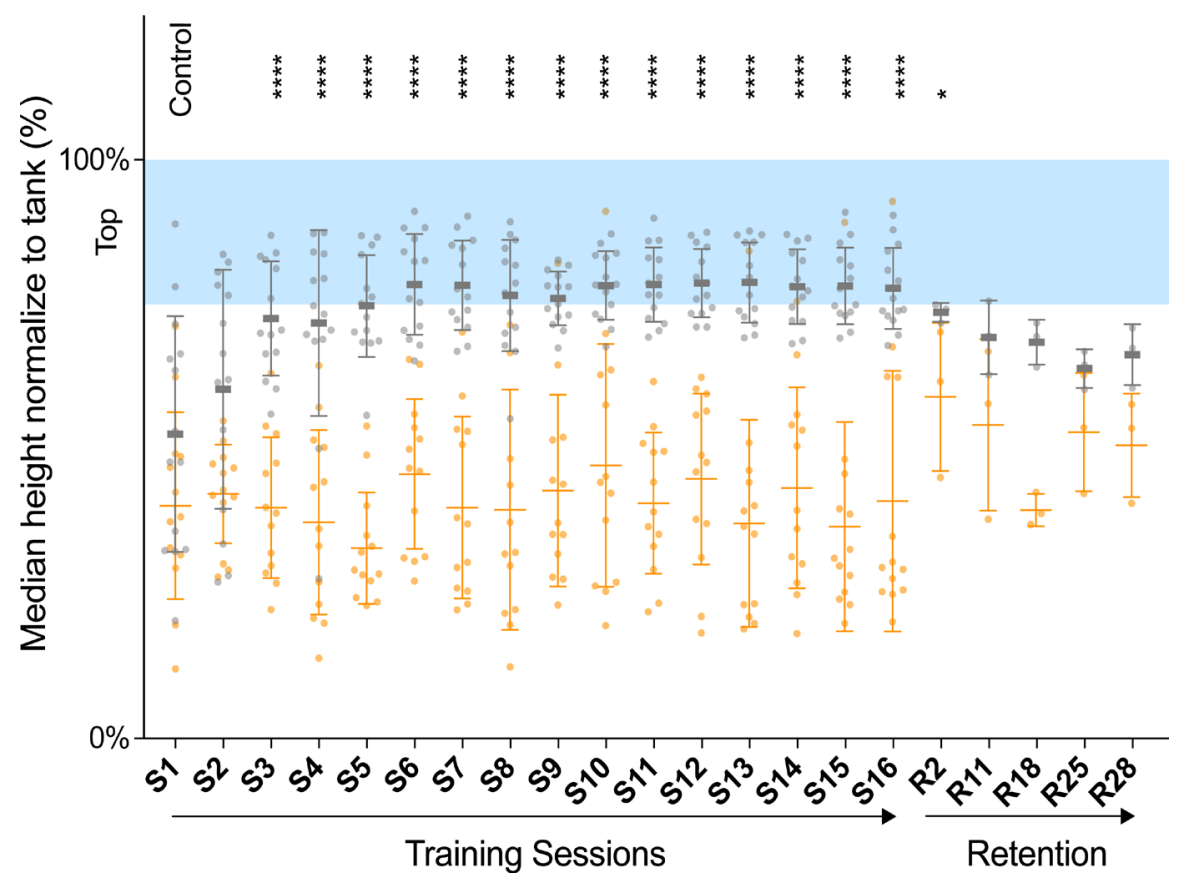

Figure 6. Median height of young fish before (yellow lines) and during (gray lines) the light stimulation. ${ }^{*}$ shows the statistical significance between $\mathrm{Y}_{\mathrm{m}}$ of a specific session with the control session $\left(\mathrm{S}_{1}\right)$ during the (light + food) stimulus where ${ }^{*},{ }^{* *},{ }^{* *}$ and ${ }^{* * * *}$ represent $\mathrm{p}<0.02, \mathrm{p}<0.01, \mathrm{p}<0.001$ and $\mathrm{p}<0.0001$ respectively. 
as eliminated stressors that could negatively impact the fish and their natural behaviours. From the researcher's perspective, the labour-intensive training and memory retention process was minimized by simultaneously training 16 tanks (each containing five fish) using identical conditions. Previous work was limited by: 1) only three tanks that could be simultaneously trained and/or tested and 2) food was localized to a very small area, which changes normal behaviour.

Behavioural analysis requires large sample sizes in order to achieve statistical significance [51]. Current zebrafish testing models are limited by a one-run sample size of only three tanks, which means that control groups and test groups are not trained at the same time [46]. This makes it difficult to identify possible inter-run variations caused by unexpected conditional changes. Unique to the high-throughput system presented in this paper is that the one-run sampling size of 16 tanks is so large that control and test groups can be concurrently trained, eliminating the effects of unexpected conditional changes. This may help to increase the statistical significance of behavioural data using fewer animals. Another benefit to a large sample group is that it may be easier to identify outliers.

Shoal behaviour was evaluated in each tank as a function of their height within the water column and was expressed as the mean height $\left(\mathrm{Y}_{\mathrm{m}}\right)$. The first exposure to the training protocol $\left(\mathrm{S}_{1}\right)$ was used to evaluate the naïve behaviour of the shoals and was compared to subsequent training sessions to determine training efficacy. The 20 seconds immediately prior to the training cue and the 20 seconds during which the training cue was provided were compared in order to ensure that the training cue was in fact triggering any observed behavioural change.

Shoals were considered trained when there was a statistically significant difference detected between $S_{1}$ and subsequent training sessions. Both the young and old fish were considered trained by $\mathrm{S}_{3}$ (Figure 5, Figure 6). From a visual inspection of the video, it was evident that the fish became more active and appeared to swim faster during the training stimulus. Based on this observation, an analysis of the average speed and the absolute average acceleration was performed, however no statistically significant values were detected and this method of analysis was not pursued further. It is thought that variations in the number of sessions required to train fish shoals are an excellent indicator of the ability of the shoal to learn. It may be possible to use this feature to determine the effect of different treatments (e.g. exposure to drugs) on the development of learning disabilities in the shoals.

Memory retention was evaluated at different days after completing the full training cycle. In this phase of the experiment, fish shoals are exposed to the light cue, without concurrent food delivery. The $\mathrm{Y}_{\mathrm{m}}$ of old fish remained statistically different from $S_{1}$ for eight days. At 14 days, $\mathrm{Y}_{\mathrm{m}}$ was no longer statistically different from $S_{1}$. $Y_{m}$ of young fish was statistically different from $S_{1}$ at two days post training. At 11 days post training $\mathrm{Y}_{\mathrm{m}}$ was not statistically different from $\mathrm{S}_{1}$. 
It is critical to note that only three shoals were tested at any one retention day and increased number of tanks may improve the statistical evaluation. Also, retention day tests on the young and old shoals were not consistent, therefore limiting the ability to make conclusions comparing the two groups. However, it can be concluded that both young and old shoals are able to remember their training for a minimum of two days. The older fish retained their training for at least 8 days. It appears that the $\mathrm{Y}_{\mathrm{m}}$ remained high at day $14\left(\mathrm{R}_{14}\right)$ for older fish (Figure 5), even though it was not statistically significant from $S_{1}$. It is expected that increasing the number of tanks at this retention time would result in a significant difference. Unfortunately, the retention of young fish was not tested between two and eight days and it is unclear exactly when they begin to forget their training. Test days for memory retention should be synchronized in future longitudinal studies when comparing young and old groups of fish. Another limitation of the set up presented was the lack of three-dimensional tracking which can potentially reveal different behavioral traits to differentiate individual fish as well as shoals from one another.

\section{Conclusions}

A novel, high-throughput system designed to analyze zebrafish behaviour with respect to learning and memory retention has been presented. This unique system provides a means to observe learning and memory retention behaviour under constant conditions using 80 fish (16 shoals of 5 fish each) simultaneously. Results confirm previous work by Doyle et al. and demonstrate the validity of the high throughput system [46] [47]. The time required to collect statistically significant data is dramatically reduced and all fish within a test group are exposed to identical conditions. This reduces the likelihood that behaviour changes would be caused by uncontrolled changes in the environment. These findings provide a foundation for understanding the normal learning and memory behaviour of zebrafish.

In future studies, it may be possible to detect changes in speed or acceleration by improving video quality. The interference between the video recording and the LEDs used to create banding artefacts could also be improved with use of a higher sampling rate video tracking system.

The system put forward provides groundwork for future studies on effects of therapeutic agents on learning and memory in a variety of brain function research including anxiety, aging, Alzheimer's disease, dementia and many others. Specifically, there is emerging evidence from researchers and the U.S. Food and Drug Administration (FDA) linking exposure to anaesthesia and the development of postoperative cognitive dysfunction in the elderly and cognitive harm in children [52] [53]. The reliable high-throughput test model presented in this paper provides a bridge for investigating this link. Given the fact that zebrafish are an excellent tool for longitudinal studies and translational research, the authors postulate that important insight into the effects of anaesthesia (specifically 
sevoflurane) on cognition in young and old groups of zebrafish can be achieved.

\section{Acknowledgements}

We would like to extend our gratitude to the Zebrafish Core Facility at Dalhousie University with a special thanks to Gretchen Wagner for her aid in breeding and upkeep of the zebrafish colonies. This research was supported by National Research Council (NRC) through Industrial Research Assistance Program (IRAP) grant 829007 and IRAP grant 868969.

\section{References}

[1] Jones, L.J., McCutcheon, J.E., Young, A.M.J. and Norton, W.H.J. (2015) Neurochemical Measurements in the Zebrafish Brain. Frontiers in Behavioral Neuroscience, 9, 1-16. https://doi.org/10.3389/fnbeh.2015.00246

[2] Gerlai, R., Chatterjee, D., Pereira, T., Sawashima, T. and Krishnannair, R. (2009) Acute and Chronic Alcohol Dose: Population Differences in Behavior and Neurochemistry of Zebrafish. Genes, Brain and Behavior, 8, 586-599.

https://doi.org/10.1111/j.1601-183X.2009.00488.x

[3] Kalueff, A.V., Echevarria, D.J. and Stewart, A.M. (2014) Gaining Translational Momentum: More Zebrafish Models for Neuroscience Research. Progress in Neuro-Psychopharmacology and Biological Psychiatry, 55, 1-6. https://doi.org/10.1016/j.pnpbp.2014.01.022

[4] Sison, M. and Gerlai, R. (2011) Associative Learning Performance Is Impaired in Zebrafish (Danio rerio) by the NMDA-R Antagonist MK-801. Neurobiology of Learning and Memory, 96, 230-237. https://doi.org/10.1016/j.nlm.2011.04.016

[5] McMenamin, S., Chandless, M. and Parichy, D.M. (2016) Working with Zebrafish at Postembryonic Stages. Methods in Cell Biology, 134, 587-607. https://doi.org/10.1016/bs.mcb.2015.12.001

[6] Stewart, A.M., Braubach, O., Spitsbergen, J., Gerlai, R. and Kalueff, A.V. (2014) Zebrafish Models for Translational Neuroscience Research: From Tank to Bedside. Trends Neurosci., 37, 264-278. https://doi.org/10.1016/j.tins.2014.02.011

[7] Valentim, A.M., Félix, L.M., Carvalho, L., Diniz, E. and Antunes, L.M. (2016) A New Anaesthetic Protocol for Adult Zebrafish (Danio rerio): Propofol Combined with Lidocaine. PLoS One, 11, 1-12. https://doi.org/10.1371/journal.pone.0147747

[8] Barbazuk, W.B., Korf, I., Kadavi, C., et al. (2000) The Synthenic Relationship of the Zebrafish and Human Genomes. Genome Research, 10, 1351-1358. https://doi.org/10.1101/gr.144700

[9] Panula, P., Sallinen, V., Sundvik, M., et al. (2006) Modulatory Neurotransmitter Systems and Behavior: Towards Zebrafish Models of Neurodegenerative Diseases. Zebrafish, 3, 235-247. https://doi.org/10.1089/zeb.2006.3.235

[10] Saif, M., Chatterjee, D., Buske, C. and Gerlai, R. (2013) Sight of Conspecific Images Induces Changes in Neurochemistry in Zebrafish. Behavioural Brain Research, 243, 294-299. https://doi.org/10.1016/j.bbr.2013.01.020

[11] Bandmann, O. and Burton, E.A. (2010) Genetic Zebrafish Models of Neurodegenerative Diseases. Neurobiology of Disease, 40, 58-65. https://doi.org/10.1016/j.nbd.2010.05.017

[12] Lieschke, G.J. and Currie, P.D. (2007) Animal Models of Human Disease: Zebrafish Swim into View. Nature Reviews Genetics, 8, 353-367. 
https://doi.org/10.1038/nrg2091

[13] Sun, Z. (2004) A Genetic Screen in Zebrafish Identifies Cilia Genes as a Principal Cause of Cystic Kidney. Development, 131, 4085-4093. https://doi.org/10.1242/dev.01240

[14] Jerman, S. and Sun, Z. (2017) Using Zebrafish to Study Kidney Development and Disease. Current Topics in Developmental Biology, 124, 41-79. https://doi.org/10.1016/bs.ctdb.2016.11.008

[15] Wilkins, B.J. and Pack, M. (2013) Zebrafish Models of Human Liver Development and Disease. Comprehensive Physiology, 3, 1213-1230. https://doi.org/10.1002/cphy.c120021

[16] Miller, N., Greene, K., Dydinski, A. and Gerlai, R. (2013) Effects of Nicotine and Alcohol on Zebrafish (Danio rerio) Shoaling. Behavioural Brain Research, 240, 192-196. https://doi.org/10.1016/j.bbr.2012.11.033

[17] Parker, M.O., Millington, M.E., Combe, F.J. and Brennan, C.H. (2012) Housing Conditions Differentially Affect Physiological and Behavioural Stress Responses of Zebrafish, as well as the Response to Anxiolytics. PLoS ONE, 7, e34992.

[18] Gerlai, R., Lee, V. and Blaser, R. (2006) Effects of Acute and Chronic Ethanol Exposure on the Behavior of Adult Zebrafish (Danio rerio). Pharmacology Biochemistry and Behavior, 85, 752-761. https://doi.org/10.1016/j.pbb.2006.11.010

[19] Miklósi, A. and Andews, R.J. (2006) The Zebrafish as a Model for Behavioral Studies. Zebrafish, 3, 227-234. https://doi.org/10.1089/zeb.2006.3.227

[20] Biggers, J.D., Finn, C.A. and McLaren, A. (1962) Long-Term Reproductive Performance of Female Mice II. Variation of Litter Size with Parity. Journal of Reproduction and Fertility, 3, 313-330.

[21] Fernandes, Y.M., Rampersad, M., Luchiari, A.C. and Gerlai, R. (2016) Associative Learning in the Multichamber Tank: A New Learning Paradigm for Zebrafish. Behavioural Brain Research, 312, 279-284. https://doi.org/10.1016/j.bbr.2016.06.038

[22] Gómez-Laplaza, L.M. and Gerlai, R. (2010) Latent Learning in Zebrafish (Danio rerio). Behavioural Brain Research, 208, 509-515.

https://doi.org/10.1016/j.bbr.2009.12.031

[23] Blaser, R.E. and Vira, D.G. (2014) Experiments on Learning in Zebrafish (Danio rerio), A Promising Model of Neurocognitive Function. Neuroscience \& Biobehavioral Reviews, 42, 224-231. https://doi.org/10.1016/j.neubiorev.2014.03.003

[24] Kalueff, A.V., Stewart, A.M., Gerlai, R. and Court, P. (2015) Zebrafish as an Emerging Model for Studying Complex Brain Disorders. Trends in Pharmacological Sciences, 35, 63-75. https://doi.org/10.1016/j.tips.2013.12.002

[25] Johnson, T.E. and Hutchinson, E.W. (1993) Absence of Strong Heterosis for Life Span and Other Life History Traits in Caenorhabditis elegans. Genetics, 134, 465-474.

[26] Al-Imari, L. and Gerlai, R. (2008) Sight of Conspecifics as Reward in Associative Learning in Zebrafish (Danio rerio). Behavioural Brain Research, 189, 216-219. https://doi.org/10.1016/j.bbr.2007.12.007

[27] Wolman, M.A., Jain, R.A., Liss, L. and Granato, M. (2011) Chemical Modulation of Memory Formation in Larval Zebrafish. Proceedings of the National Academy of Sciences of the United States of America, 108, 15468-15473. https://doi.org/10.1073/pnas.1107156108

[28] Hinz, F.I., Aizenberg, M., Tushev, G. and Schuman, E.M. (2013) Protein Synthesis-Dependent Associative Long-Term Memory in Larval Zebrafish. Journal of 
Neuroscience, 33, 15382-15387. https://doi.org/10.1523/JNEUROSCI.0560-13.2013

[29] Blank, M., Guerim, L.D., Cordeiro, R.F. and Vianna, M.R.M. (2009) A One-Trial Inhibitory Avoidance Task to Zebrafish: Rapid Acquisition of an NMDA-Dependent Long-Term Memory. Neurobiology of Learning and Memory, 92, 529-534. https://doi.org/10.1016/j.nlm.2009.07.001

[30] Braubach, O.R., Wood, H.D., Gadbois, S., Fine, A. and Croll, R.P. (2009) Olfactory Conditioning in the Zebrafish (Danio rerio). Behavioural Brain Research, 198, 190-198. https://doi.org/10.1016/j.bbr.2008.10.044

[31] Mathur, P., Lau, B. and Guo, S. (2011) Conditioned Place Preference Behavior in Zebrafish. Nature Protocols, 6, 338-345. https://doi.org/10.1038/nprot.2010.201

[32] Del Bene, F., Wyart, C., Robles, E., et al. (2011) Filtering of Visual Information in the Tectum by an Identified Neural Circuit. Science, 330, 669-673. https://doi.org/10.1126/science.1192949

[33] Wyart, C., Del Bene, F., Warp, E., et al. (2009) Optogenetic Dissection of a Behavioural Module in the Vertebrate Spinal Cord. Nature, 461, 407-410.

https://doi.org/10.1038/nature08323

[34] Fetcho, J.R. and McLean, D.L. (2010) Some Principles of Organization of Spinal Neurons Underlying Locomotion in Zebrafish and Their Implications. Annals of the New York Academy of Sciences, 1198, 94-104. https://doi.org/10.1111/j.1749-6632.2010.05539.x

[35] Valente, A., Huang, K.-H., Portugues, R. and Engert, F. (2012) Ontogeny of Classical and Operant Learning Behaviors in Zebrafish. Learning \& Memory, 19, 170-177. https://doi.org/10.1101/lm.025668.112

[36] Spence, R., Gerlach, G., Lawrence, C. and Smith, C. (2008) The Behaviour and Ecology of the Zebrafish Danio rerio. Biological Reviews, 83, 13-34. https://doi.org/10.1111/j.1469-185X.2007.00030.x

[37] Gleason, P.E., Weber, P.G. and Weber, S.P. (1977) Effect of Group Size on Avoidance Learning in Zebra Fish, Brachy Danio rerio (Pisces: Cyprinidae). Animal Learning \& Behavior, 5, 213-216. https://doi.org/10.3758/BF03214081

[38] Vital, C. and Martins, E.P. (2013) Socially-Central Zebrafish Influence Group Behavior More than Those on the Social Periphery. PLOS ONE, 8, e55503. https://doi.org/10.1371/journal.pone.0055503

[39] Griffin, G. (2012) Evaluating Environmental Enrichment Is Essential. Enrichment Record, 29-33.

[40] Kistler, C., Hegglin, D., Würbel, H. and König, B. (2011) Preference for Structured Environment in Zebrafish (Danio rerio) and Checker Barbs (Puntius oligolepis). Applied Animal Behaviour Science, 135, 318-327. https://doi.org/10.1016/j.applanim.2011.10.014

[41] Reed, B. and Jennings, M. (2011) Guidance on the Housing and Care of Zebrafish Danio rerio. Res. Anim. Dep. Sci. Group, RSPCA, 1-27.

[42] Yeh, C.M., Glöck, M. and Ryu, S. (2013) An Optimized Whole-Body Cortisol Quantification Method for Assessing Stress Levels in Larval Zebrafish. PLoS ONE, 8, e79406. https://doi.org/10.1371/journal.pone.0079406

[43] Pavlidis, M., Digka, N., Theodoridi, A., et al. (2013) Husbandry of Zebrafish, Danio rerio, and the Cortisol Stress Response. Zebrafish, 10, 524-531. https://doi.org/10.1089/zeb.2012.0819

[44] Ramsay, J.M., Fiest, G.W., Varga, Z.M., Westerfield, M., Kent, M.A. and Schreck, C.B. (2015) Whole-Body Cortisol Response of Zebrafish to Acute Net Handling 
Stress. Aquaculture, 297, 157-162. https://doi.org/10.1016/j.aquaculture.2009.08.035

[45] Southwell, M., Galassi, M. and McRobert, S. (2012) Fish Cam: An Online Tool for Introducing Shoaling Behavior to the Classroom. Zebrafish, 9, 242-245. https://doi.org/10.1089/zeb.2012.0755

[46] Doyle, J.M., Merovitch, N., Wyeth, R.C., et al. (2017) A Simple Automated System for Appetitive Conditioning of Zebrafish in Their Home Tanks. Behavioural Brain Research, 317, 444-452. https://doi.org/10.1016/j.bbr.2016.09.044

[47] Wyeth, R.C., Braubach, O.R., Fine, A. and Croll, R.P. (2010) Videograms: A Method for Repeatable Unbiased Quantitative Behavioral Analysis without Scoring or Tracking CO. Neuromethods, 51, 15-33.

[48] Suriyampola, P.S., Sykes, D.J., Khemka, A., Shelton, D.S., Bhat, A. and Martins, E.P. (2016) Water Flow Impacts Group Behavior in Zebrafish (Danio rerio). Behavioral Ecology, 1-7.

[49] Care CC on A (1993) Guide to the Care and Use of Experimental Animals. Vol. 1, 2nd Edition, Canadian Council on Animal Care, Ottawa.

[50] Branson, K., Robie, A., Bender, J., Perona, P. and Dickinson, M. (2009) High-Throughput Ethomics in Large Groups of Drosophila. Nature Methods, 6, 451-457. https://doi.org/10.1038/nmeth.1328

[51] Taborsky, M. (2010) Sample Size in the Study of Behaviour. Ethology, 116, 185-202. https://doi.org/10.1111/j.1439-0310.2010.01751.x

[52] SmartTots. Funding Anesthesia Research to Ensure Pediatric Safety. http://smarttots.org/

[53] The Effect of General Anesthesia on the Developing Brain: Appreciating Parent Concerns while Allaying Their Fears.

http://www.apsf.org/newsletters/html/2016/Oct/04_DevBrain.htm 


\section{Appendix}

\section{A.1. Background}

Due to horizontal banding artefacts from the LED lighting in the recordings, the background of each video had to be accurately determined. After the trimming of the pre- and mid-light videos, separate backgrounds for each condition were generated using an average background of $80 \%$ to $100 \%$ of the total number of frames.

\section{A.2. Shape Bounds}

Minimum, maximum, and mean areas for the body of zebrafish in the video analysis were manually set, as determined by trial and error, in order to minimise false positives in the detection and tracking the fish.

\section{A.3. Jump Distance}

Jump distances in the previous and current position of the fish were determined through trial and error. The maximum distance between predicted position and observed position of a subject to be matched was found to be 10.0 pixels. The maximum distance between the position of predicted and observed subjects from a blob of two or more subjects was found to be 100.0 pixels. The minimum distance between the previous and current centre position to consider the fish jumping as predicted was determined to be 100.0 pixels.

\section{A.4. Angle Dampening}

The change in predicted position with regard to the amount by which a change in the direction of the movement of the zebrafish will alter the velocity of its movement was determined to be 0.5 in order to minimise errors in tracking.

\section{A.5. Observation Parameters}

\section{Max Area Delete}

The maximum area of a detected component that cannot be merged with any other components for the component to be ignored was found to be 0.1 pixels.

\section{Min Area Ignore}

The minimum area for large foreground detections that may be caused by objects other than fish or lighting changes to be ignored was found to be 1.0 pixels.

\section{Max Penalty Merge}

The maximum total distance between a subject and pixels labeled as background for those two objects to be merged was found to be 100.0 pixels in order to minimise tracking errors.

\section{Max Clusters per Blob}

The maximum number of clusters into which a component can be split during forward tracking was found to be 1 .

\section{Max Blobs to detect}

Since there were a total of five fish in each tank, this was set as the maximum 
number of objects to be detected in the foreground. In the event that a greater number of fish were detected, the five largest objects detected by the program were used to predict each position.

\section{A.6. Hindsight}

Detections that were mistakenly split into two observations were minimised by merger (if the merge penalty was less than 500.00 pixels) of the observations for up to 500 frames. Any objects detected with a lifespan of less than 1 frame were deleted. 